\title{
Effect of Covid-19 Vaccines Against Delta Variant. A Review Article
}

\author{
Mohamed Raslan², Eslam MS ${ }^{2}$, Sara $\mathrm{AR}^{2}$ and Nagwa A Sabri ${ }^{1 *}$ \\ ${ }^{1}$ Department of Clinical Pharmacy, Faculty of Pharmacy, Ain Shams University, \\ Cairo, Egypt \\ ${ }^{2}$ Drug Research Centre, Cairo, Egypt \\ *Corresponding Author: Nagwa A Sabri, Department of Clinical Pharmacy, Faculty \\ of Pharmacy- Ain Shams University, Cairo, Egypt.
}

Received: December 06, 2021

Published: January 10, 2022

(C) All rights are reserved by Nagwa A Sabri., et al.

\begin{abstract}
Background: Coronavirus Disease-2019 is a novel coronavirus strain that is producing an infection that is quickly spreading over the world. Several waves of the disease occurred and different variants were discovered.

Aim: To investigate the potential threat of emerging COVID-19 new delta variants, and their effect on vaccination efficacy, hospitalization rates, and mortality rates.

Discussion: Studies indicated covid-19 patients infected with the delta strain were more likely to be hospitalized or seek emergency treatment than those infected with the alpha type. According to the findings, outbreaks of the delta variation among unvaccinated populations may exert a greater pressure on health-care systems than epidemics of the alpha type. Furthermore, it was estimate that the hazard of death related to B.1.1.7 variant infection is $6 \%$ greater than with pre-existing variants. Besides, the B.1.1.7 type is not only more contagious than pre-existing covid-19 strains, but it may also cause more severe illness. More research findings indicated that the chances of vaccine infection breakthrough were much higher in delta variant infections compared to alpha strain cases, suggesting that mRNA vaccines were less effective in preventing infection with the delta type.

Conclusion: It can be concluded that the delta variation (B.1.617.2) impairs the efficacy of critical therapeutic monoclonal antibodies for COVID-19, raises the absolute risk of death, particularly in the elderly, reduces immunization efficacy, and increases the likelihood of hospitalization.
\end{abstract}

Keywords: COVID-19; Delta Variant; Alpha Variant; B.1.617.2; Bnt162b2 Vaccine; Chadox1 Ncov-19 Vaccine

\section{Introduction}

Coronavirus Disease-2019 (COVID-19) is a new strain of coronaviruses that is causing an infection that has rapidly spread all over the world. The primary pathways of infection spreading reported to be through large respiratory droplets, and disease severity ranging from mild self-limiting flu-like illness to acute pneumonia, respiratory collapse, and death. Patients with serious mental illnesses and depressive disorders are more vulnerable to COVID-19 infection, which can be attributed to a variety of factors such as impaired cognitive functions, poor awareness, and noncompliance with infection control measures [1].
After a year and a half of the Coronavirus Disease 2019 (COVID-19) pandemic, we have reached the heartbreaking milestone of four million COVID-19 fatalities. Estimates of the real number of fatalities based on extra deaths range over ten million [2].

The real number of cases is believed to be five to twenty times greater than the number of verified cases, reaching billions. Fortunately, we have many effective vaccinations at our disposal that may be able to control the COVID-19 epidemic. Unfortunately, our immunization efforts must cope with rapidly spreading Severe Acute Respiratory Syndrome Coronavirus 2 (SARS-CoV-2) strains, which presents a massive obstacle [3]. 
While the number of vaccinated people globally has surpassed one billion, new variants of concern have appeared that are more worrying than their predecessors, with the Delta variant being the most concerning so far. The variants are worrisome because they possess enhanced transmissibility, increased illness severity, and immunological evasion, increasing the likelihood of reinfections in the elderly or breakthrough infections in the vaccinated [4].

The SARS-CoV-2 Delta variant (B.1.617.2) has been found in a number of countries. It has grown prevalent in the Indian state of Maharashtra and, most likely, other Indian regions, accounting for $77 \%$ of the sequenced viruses disseminated in the UK between June 2 and June 9, 2021. It has been designated as a variation of concern and is thought to be $60 \%$ more transmissible than the Alpha variant (B.1.1.7). Little is known about the Delta variant's susceptibility to the humoral immune response. According to recent research, individuals of the B.1.617 lineage are less sensitive to some monoclonal and polyclonal antibodies than the alpha variant [5].

Coronavirus SARS-CoV-2 enters the cell via ACE2 receptor. Furthermore, ACE2 is highly expressed in type II alveolar cells in the lungs, where also ApoE is one of the highly co-expressed genes. Investigations showed that ApoE was associated with higher prevalence and mortality of COVID-19 in individuals with genotype ApoEe4 [6].

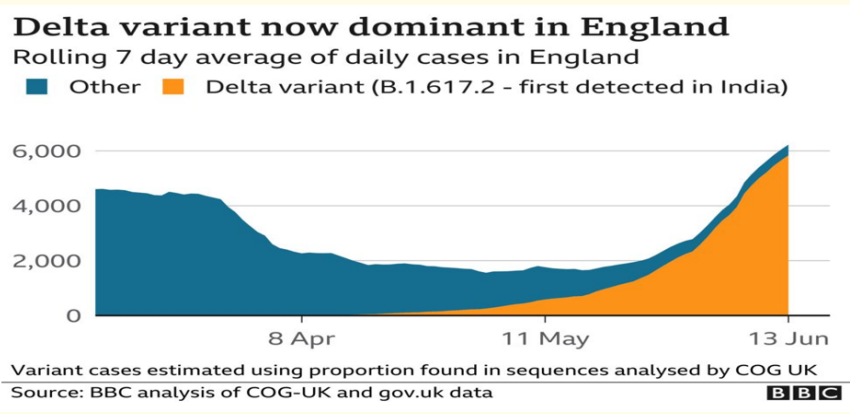

Figure 1: Numbers of Delta variant COVID-19 infection in United Kingdom [7].

The sites of the spike protein mutations in the Delta variant were found to be comparable to those seen in other variants of concern. In particular, the D950N mutation, like the D614G mutation, localized to the trimer interface, indicating that this mutation, like the D614G mutation, may contribute to the control of spike protein dynamics. Some mutations in the Delta variant cluster in the NTD. The Delta variant's 156-157 deletion and G158R mutation map to the same surface as the Alpha and Beta (B.1.351) variants' 144 and 241-243 deletions, respectively. The T19R mutation corresponds to a surface region in the Alpha variant that contains multiple mutations. These modified residues are located in the NTD 'supersite,' which is targeted by the majority of anti-NTD neutralizing antibodies $[8,9]$.

In the RBD, mutations found in concern variants localize to the ACE2-binding surface's periphery, indicating that the virus accumulates mutations there to decrease or evade antibody recognition while retaining ACE2 binding. For example, the L452R mutation in the Delta variation, which is situated in the periphery, affects antibody neutralization. The single mutation inside the ACE2 patch is at position 501, which enhances the RBD's affinity for ACE2 and also plays a role in antibody escape. The T478K mutation in the RBD is specific to the Delta variant and lies inside the epitope area of Class 1 powerful neutralizing monoclonal antibodies. This mutation is similar to the E484K mutation, which allows antibodies to escape. These findings encouraged us to investigate the neutralizing potential of monoclonal antibodies and sera from people who had recovered from COVID-19 and those who had been vaccinated against COVID-19 against the Delta variant $[5,10,11]$.

\section{Discussion}

A cohort analysis was conducted among all COVID-19 patients in England between March 29 and May 23, 2021, who were found to be infected with either the alpha or delta SARS-CoV-2 variants. Individual-level data on these patients were connected to regular health-care databases on immunization, emergency attendance, hospitalization, and death. The risk of hospitalization and emergency care attendance was evaluated throughout the entire population and by vaccination status subgroups in individuals with sequencing-confirmed delta and alpha mutations [12].

Individual-level data on 43,338 COVID-19-positive individuals (8682 with the delta variant, 34,656 with the alpha variant; median age 31 years) were analyzed. Within 14 days of receiving the specimen, 2.3 percent of patients with the delta variant and 2.2 percent of patients with the alpha variant were admitted to the hospital. 5.7 percent of patients with the delta variation and 4.2 
percent of individuals with the alpha variant were admitted to the hospital or sought emergency treatment. Most patients were unvaccinated (74.0\% across both groups). The HRs for vaccinated patients with the delta variant versus the alpha variant (adjusted HR for hospital admission 1.94 and for hospital admission or emergency care attendance 1.58) were similar to the HRs for unvaccinated patients ( 2.32 and $1.43 ; \mathrm{p}=0.82$ for both) but the precision for the vaccinated subgroup was low. According to the study's interpretation, individuals with COVID-19 infected with the delta variant had a greater chance of hospitalization or emergency care attendance than those infected with the alpha variant. The findings indicate that outbreaks of the delta variant in unvaccinated populations may place a higher strain on health-care systems than outbreaks of the alpha variant [13].

Several studies have found that B.1.1.7 is more transmissible than pre-existing variations, although none have found that it affects disease severity. A dataset was examined that linked 2,245,263 positive SARS-CoV-2 cases and 17,452 fatalities related with COVID-19 in England between 1 November 2020 and 14 February 2021. The existence or absence of B.1.1.7 can be determined in $51 \%$ of these cases because mutations in this lineage impede PCR amplification of the spike (S) gene target (also known as $\mathrm{S}$ gene target failure) (SGTF). Based on 4,945 fatalities with known SGTF status, we estimate that the hazard of mortality associated with SGTF is 55\% greater than in instances without SGTF after controlling for age, gender, ethnicity, deprivation, care home residence, local authority of domicile, and test date. This match with the fact that within 28 days of a positive test in the community, the absolute risk of mortality for a 55-69-year-old male rises from 0.6 percent to 0.9 percent. We estimate that the hazard of mortality associated with B.1.1.7 is $61 \%$ greater than with pre-existing variants after correcting for SGTF misclassification and missingness in SGTF status. According to our findings, B.1.1.7 is not only more contagious than pre-existing SARS-CoV-2 variants, but it may also induce more severe disease [14].

In vitro studies revealed that B.1.617.2 is roughly 6-fold less susceptible to neutralizing antibodies in recovered people' sera and approximately 8-fold less sensitive to vaccine-elicited antibodies than wild type Wuhan-1 with D614G. (B.1.617.2 spike) pseudo typed viruses were shown to be less sensitive to therapeutically authorized monoclonal antibodies against the receptor binding do-

\section{Pfizer Vaccine Losing Efficacy Faster Against Delta Variant}

Effectiveness of COVID-19 vaccines against infections with a high viral load variant* over time (in percent) Months after second vaccine shot: 미 $1=3$ $-12$ Efficacy loss
(percentage points)

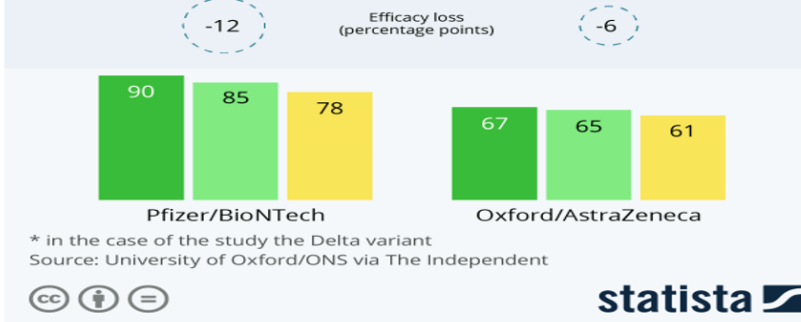

Figure 2: Statistical diagram showing compensated vaccine efficacy against delta variant covid-19 infection by time. The diagram describes the loss in vaccination efficacy of (Pfizer/ BioNTech) and (Oxford/Astrazeneca) over 1, 2, and 3 months after the second vaccine shot [15].

main (RBD) and N-terminal domain (NTD), namely bamvalinumb and imdevimab monoclonal antibodies [16].

In addition, the delta variant (B.1.617.2) showed increased replication efficiency in both airway organoid and human airway epithelial systems than B.1.1.7, which was linked with the B.1.617.2 spike being preferentially cleaved compared to B.1.1.7. In a study of vaccinated healthcare workers from three sites in India during a period of mixed lineage circulation, we found that ChAdOx-1 vaccination effectiveness against B.1.617.2 was lower than that of non-B.1.617.2. These epidemiological and in vitro results suggest that B.1.617.2's dominance in India is most likely due to a combination of evasion of neutralizing antibodies in previously infected people and enhanced viral infectivity. Delta variant compromises the efficacy of essential therapeutic monoclonal antibodies for COVID-19, and reduced vaccine efficacy necessitates post-vaccination infection control measures [17] like face mask, face shields, social distancing, medical gloves, herbs, and healthy diet [18].

The efficacy of the BNT162b2 and ChAdOx1 nCoV-19 vaccines against this variant is unknown. Research utilized a test-negative case-control design to evaluate the efficacy of immunization against symptomatic illness caused by the delta variation or the prevalent alpha strain (B.1.1.7) during the period when the delta 
variant was first discovered. Sequencing was used to identify variants, which were based on the spike (S) gene status. Data from all symptomatic sequencing Covid-19 cases in England were used to estimate the proportion of cases with either variation based on the patients' vaccination status [19].

According to the study findings, effectiveness after one dose of vaccination (BNT162b2 or ChAdOx1 $\mathrm{nCoV}-19$ ) was significantly lower in people with the delta variation (30.7 percent) than in people with the alpha variant (48.7 percent); the results were comparable for both vaccines. The efficacy of two doses of the BNT162b2 vaccination was 93.7 percent in people with the alpha variation and 88.0 percent in people with the delta version. The efficacy of two doses of the ChAdOx1 $\mathrm{nCoV}-19$ vaccine was 74.5 percent in people with the alpha variant and 67.0 percent in people with the delta variant. After receiving two vaccination doses, the observed findings show very minor variations in vaccine efficacy between the delta and alpha variants. Absolute disparities in vaccination efficacy became more pronounced after the first dosage. This finding would provide credence to efforts to increase vaccination uptake among vulnerable groups by administering two doses [20].

In a surrogate test against the SARS-CoV-2 variants of concern alpha, beta, and gamma, heterologous boosting with BNT162b2 was found to produce larger numbers of spike-specific $\mathrm{CD}_{4+}$ and $\mathrm{CD}_{8+}$ T cells, as well as high titers of neutralizing antibodies [21].

The rapid development of the delta form, on the other hand, poses a concern for both ChAdOx1-S-primed vaccinations anticipating a boost vaccination and those who have already been fully vaccinated with ChAdOx1-S. A study analyzed plasma from ChAdOx1S-primed vaccines at a mean 16.3 days (range 14-22 days) after homologous ChAdOx1-S (group $1 ; n=12$, seven women) or heterologous BNT162b2 (group 2; $n=11$, eight women) boost. The mean dose interval between prime and boost was 73.5 days (range 71-85 days) and did not differ between the groups [22].

Mean anti-spike IgG was 171.9 relative units (RU) per mL (SD $121 \cdot 8 \mathrm{RU} / \mathrm{mL}$ ) in group 1 and $611.0 \mathrm{RU} / \mathrm{mL}$ (SD 104.5 RU/mL) in group 2 ( $\mathrm{p}<0 \cdot 0001)$. Individuals in Group 1 showed a moderate $50 \%$ neutralization titer (NT50) against the wild type and alpha variant, and this activity was even lower against beta, gamma, and delta variants. All heterologous ChAdOx1-S/BNT162b2 vaccinated people, on the other hand, obtained at least NT50 $=25$ against all variations, including the delta variant (NT50 $\geq 100$ in 85 percent of vaccinations). In both groups, mean anti-spike IgG associated strongly with NT50 against the delta variant $(r=0.901 ; p<0.0001)$. The effective suppression of variations, particularly the delta variant, lends credence to heterologous ChAdOx1-S/BNT162b2 vaccination. If validated in larger research, our findings suggest a heterologous boost vaccination of individuals who have already received homologous ChAdOx1-S immunization, once humoral immunity has waned and patients are vulnerable to infection [23].

As part of clinical care, patients had serial COVID-19 RT-PCR, blood testing for serology, acute phase reactants, and chest imaging. The patient was questioned for a clinical history, and reports and case documents were obtained. From the patient>s breakthrough infections and the assumed index case, stored RT-PCR positive samples were recovered for whole genome sequencing of SARSCoV-2. According to the data, the patient had three RT-PCR confirmed SARS-CoV-2 infections. Two breakthrough infections occurred within three weeks of each other, with the first occurring more than three weeks after full immunization and despite postvaccination seroconversion [24].

The Alpha form caused the first breakthrough infection, while the Delta form caused the second. The Delta variant infection led in hypoxia, hospitalization, and a seven-week sickness. Despite previous infection, full immunization, and seroconversion, it was determined that the patient had an Alpha variant breakthrough infection. Despite boosting following this infection, the patient went on to have a severe Delta variant breakout infection. The illness was passed down from a completely vaccinated family member to the patient [25].

Researchers conducted case research to assess the efficacy of mRNA vaccinations against Delta (B.1.617.2) vs Alpha (B.1.1.7) strains. RT-PCR positive cases reported to the National Surveillance System between the 17th of May and the 4th of July 2021 (weeks 20 to 26), as well as demographic and vaccination status information obtained from the electronic vaccination registry, were utilized [26].

SARS-CoV-2 variants were classified using whole-genome sequencing (WGS) or spike (S) gene target failure (SGTF) data. The odds of vaccinated persons becoming infected (odds of vaccine infection breakthrough) in Delta SARS-CoV-2 cases compared to 
Alpha SARS-CoV-2 cases were calculated using conditional logistic regression, which was adjusted for age group, gender, and week of diagnosis [27].

Individuals infected with the Delta strain were immunized at a higher rate $(12 \%)$ than those infected with the alpha variant (5\%). When comparing Delta cases to Alpha cases, there was a statistically significant greater chances of vaccine infection breakthrough for partial ( $\mathrm{OR}=1.70)$ and complete vaccination ( $\mathrm{OR}=1.96)$, implying reduced mRNA vaccine efficacy against Delta cases. In the secondary analysis, it was found that the Delta form patients had lower mean cycle threshold (Ct) values than the Alpha variant, independent of vaccination status. Furthermore, the Delta variant cases exhibited a Ct-value mean rise of 2.24 between unvaccinated and fully vaccinated breakthrough cases, compared to 4.49 in the $\mathrm{Al}-$ pha variant, indicating that the vaccination had a smaller influence on viral load in Delta cases. Study conclusions revealed that the probabilities of vaccine infection breakthrough were substantially greater in Delta cases compared to Alpha cases, suggesting that the mRNA vaccinations were less efficient in preventing infection with the Delta type. Furthermore, the vaccination breakthrough cases are expected to have higher mean Ct values, implying greater infectiousness with the Delta variant infection [28].

Using sera from patients vaccinated with BNT162b2, a substantial reduction in neutralizing antibody titer was observed for B.1.617.2 compared to B.1.1.7. After two doses of BNT162b2 or AZD1222, respectively, vaccine efficacy of 88 percent or 67 percent against symptomatic illness following infection with B.1.617.2 was reported in England. However, it was not feasible to evaluate the vaccine's efficacy against severe illness since there were few severe cases throughout the research period of April to June 2021. Although both the AZD1222 and BNT162b2 vaccines were successful in lowering the risk of infection and hospitalization due to B.1.617.2 in Scotland, the degree of protection was not as great as against B.1.1.7 [29].

A research study evaluated the efficacy of two full-length Spike protein-encoding mRNA vaccines from Moderna (mRNA-1273) and Pfizer/BioNTech (BNT162b2) in the Mayo Clinic Health System from January to July 2021, while either the Alpha or Delta variation was common. We created cohorts of vaccinated and unvaccinated Minnesota residents $(n=25,589$ each) who were matched by age, gender, race, history of past SARS-CoV-2 PCR testing, and date of complete vaccination. During the research period, both vaccinations were very effective against SARS-CoV-2 infection (mRNA-
1273: 86\%, BNT162b2: 76\%) and COVID-19-related hospitalization (mRNA-1273: 91.6 percent, BNT162b2: 85 percent). However, in July, the efficiency of mRNA-1273 against infection was significantly reduced (76 percent), with an even greater decline in effectiveness for BNT162b2 (42 percent) [20].

Notably, the frequency of the Delta form in Minnesota grew from 0.7 percent in May to more than 70 percent in July, whereas the prevalence of the Alpha variant fell from 85 percent to 13 percent over the same time period. When rates of infection were evaluated between matched persons completely vaccinated with mRNA1273 vs BNT162b2 throughout different states, mRNA-1273 imparted a two-fold risk reduction against breakthrough infection (IRR = 0.50). In Florida, which is presently suffering its most severe COVID-19 outbreak to date, the risk of infection following full immunization with mRNA-1273 was approximately $60 \%$ lower than after full vaccination with BNT162b2 (IRR: 0.39) [30].

\section{Conclusion}

We can clearly conclude that delta variant (B.1.617.2) compromises the efficacy of essential therapeutic monoclonal antibodies for COVID-19, increases the absolute risk of mortality especially for geriatrics, and reduce vaccination efficacy, and raise the chance of hospitalization need. That can increase the burden on healthcare facilities. This will necessitate a strict post-vaccination infection control measures.

\section{Bibliography}

1. Nagwa Ali Sabri., et al. "Depressive Disorders and Incidence of COVID-19: Is There a Correlation and Management Interference?" Psychological Disorders and Research 3.2 (2019): 2-7.

2. "There have been $7 \mathrm{~m}-13 \mathrm{~m}$ excess deaths worldwide during the pandemic". The Economist (2021).

3. Noh J and Danuser G. "Estimation of the fraction of COVID-19 infected people in US states and countries worldwide". PLoS ONE 16 (2021): e0246772.

4. Tracking SARS-CoV-2 variants (2021).

5. Planas D., et al. "Reduced sensitivity of SARS-CoV-2 variant Delta to antibody neutralization". Nature 596.7871 (2021): 276-280.

6. Sabri NA., et al. "Association of COVID-19 with genotyping ApoEe4 and APOBEC3B". Allied Journal of Medical Research 4.1 (2020): 55-56. 
7. Rachel Schraer. "Covid: Why has the Delta variant spread so quickly in UK?" BBC News (2021).

8. Plante JA., et al. "The variant gambit: COVID-19's next move". Cell Host and Microbe 29 (2021): 508-515.

9. McCallum M., et al. "N-terminal domain antigenic mapping reveals a site of vulnerability for SARS-CoV-2". Cell 184 (2021): 2332-2347.

10. Starr TN., et al. "Complete map of SARS-CoV-2 RBD mutations that escape the monoclonal antibody LY-CoV555 and its cocktail with LY-CoV016". Cell Reports Medicine 2 (2021): 100255 (2021).

11. Barnes CO., et al. "SARS-CoV-2 neutralizing antibody structures inform therapeutic strategies". Nature 588 (2020): 682-687.

12. Connor TR., et al. "CLIMB (the Cloud Infrastructure for Microbial Bioinformatics): an online resource for the medical microbiology community". Microbial Genome (2016): 2e000086.

13. Twohig., et al. "Hospital admission and emergency care attendance risk for SARS-CoV-2 delta (B.1.617.2) compared with alpha (B.1.1.7) variants of concern: a cohort study". The Lancet Infectious Diseases (2021).

14. Davies NG and Jarvis CI. "CMMID COVID-19 Working Group. et al. Increased mortality in community-tested cases of SARSCoV-2 lineage B.1.1.7". Nature 593 (2021): 270-274.

15. Katharina Buchholz. COVID-19 Vaccines "Pfizer Losing Efficacy Faster Against Delta Variant". Statista (2021).

16. Weinreich DM., et al. "REGN-COV2, a Neutralizing Antibody Cocktail, in Outpatients with Covid-19". The New England Journal of Medicine 384 (2021): 238-251.

17. Petra Mlcochova., et al. "SARS-CoV-2 B.1.617.2 Delta variant emergence, replication and sensitivity to neutralising antibodies". bioRxiv (2021): 443253.

18. Nagwa A Sabri., et al. "Impact of Applied Protective Measures of COVID-19 on Public Health". Acta Scientific Pharmaceutical Sciences 5.7 (2021): 63-72.

19. Public Health England. "SARS-CoV-2 variants of concern and variants under investigation in England" (2021).

20. Lopez Bernal J., et al. "Effectiveness of Covid-19 Vaccines against the B.1.617.2 (Delta) Variant”. The New England Journal of Medicine 385.7 (2021): 585-594.

21. Barros-Martins J., et al. "Immune responses against SARSCoV-2 variants after heterologous and homologous ChAdOx1 nCoV-19/BNT162b2 vaccination". Nat Med 27.9 (2021): 15251529.
22. Arora P., et al. "Increased lung cell entry of B.1.617.2 and evasion of antibodies induced by infection and BNT162b2 vaccination". bioRxiv (2021).

23. Behrens Georg MN., et al. "SARS-CoV-2 delta variant neutralisation after heterologous ChAdOx1-S/BNT162b2 vaccination". The Lancet 398.10305 (2021): 1041-1042.

24. Shastri Jayanthi., et al. "Severe SARS-CoV-2 Breakthrough Reinfection with Delta Variant After Recovery from Breakthrough Infection by Alpha Variant in a Fully Vaccinated Health Worker". Frontiers in Medicine 8 (2021): 1379.

25. Mlcochova P., et al. "SARS-CoV-2 B.1.617.2 Delta variant emergence and vaccine breakthrough". bioRxiv Preprint (2021).

26. National Institute of Health (INSA) "Genetic diversity of the novel coronavirus SARS-CoV-2 (COVID-19) in Portugal". Lisbon (2021).

27. Borges V., et al. "Tracking SARS-CoV-2 lineage B.1.1.7 dissemination: insights from nationwide spike gene target failure (SGTF) and spike gene late detection (SGTL) data, Portugal, week 492020 to week 3 2021". Eurosurveillance 26 (2021): 1-6.

28. Irina Kislaya., et al. "Delta variant and mRNA Covid-19 vaccines effectiveness: higher odds of vaccine infection breakthroughs". MedRxiv (2021).

29. Tregoning JS., et al. "Progress of the COVID-19 vaccine effort: viruses, vaccines and variants versus efficacy, effectiveness and escape". Nature Reviews Immunology (2021).

30. Arjun Puranik., et al. "Comparison of two highly-effective mRNA vaccines for COVID-19 during periods of Alpha and Delta variant prevalence". Medrxiv (2021).

\section{Assets from publication with us}

- Prompt Acknowledgement after receiving the article

- Thorough Double blinded peer review

- Rapid Publication

- Issue of Publication Certificate

- High visibility of your Published work

Website: www.actascientific.com/

Submit Article: www.actascientific.com/submission.php

Email us: editor@actascientific.com

Contact us: +919182824667 